\title{
Preparing for the vaccine
}

\author{
Clear and consistent messaging on COVID-19 vaccine rollout will be key to building public trust and managing \\ expectations.
}

A s approval of the first COVID-19 vaccine draws closer, attention is shifting to how to manufacture and distribute sufficient shots to supply national or global immunization programs. The challenge is unprecedented: to make enough vaccine not for a few million people, but for hundreds of millions of people. Ironically, the RNA and viral vector vaccines that are furthest along in clinical development are the least suitable for large-scale manufacture, storage and distribution. Until conventional vaccines become available that can capitalize on the existing immunization and distribution infrastructure, it seems likely that a large proportion of the world's population will remain unvaccinated in 2021. In this context, clear and consistent public health communication to manage expectations about vaccine distribution, who gets the vaccine first, and full transparency as to the benefits as well as the risks will be key to building trust.

Thus far, public health messaging during the epidemic has been anything but clear and consistent.

Part of the problem is that new research has regularly upended expert advice, with inconsistent messaging on masks, testing and social distancing that has confused public understanding and compliance.

Vaccines represent an intervention where effective and consistent messaging will be critical to success because in recent decades scientific consensus on their value has been recast as controversy (or even conspiracy) in the public sphere. Social media have reinforced vaccine hesitancy, with religious leaders, family members or other trusted sources often perpetuating myths and misinformation.

According to a September report from Pew Research, only $51 \%$ of US adults say they definitely or probably would get vaccinated with a COVID-19 vaccine - a $21 \%$ percentage point drop from May. A survey of Europeans by the Vaccine Confidence Project found that $20 \%$ of Swiss and 18\% of French respondents would refuse a vaccine; similarly, a recent YouGov survey found that one in six British adults would definitely or probably turn down a shot.

But ironically, it may be the unrealistic expectations of vaccine proponents that turns out to be as much as a problem: $55 \%$ of them would, apparently, be somewhat dissuaded if the vaccine was "only" $60 \%$ effective. And yet a vaccine that halves the risk of infection for an individual would be sufficient for a US Food and Drug Administration Emergency Use Authorization (EUA). For a public that expected $100 \%$ efficacy, $50 \%$ might feel like failure.

For SARS-CoV-2, many unanswered questions remain about natural immunity to the virus, let alone the nature and length of immune protection afforded by a vaccine. As long as doubts remain about the frequency of repeated infections, the capacity of reinfected individuals to spread or shed virus as well as develop symptomatic or even serious disease, and the length of protection afforded by neutralizing antibody titers, doubts will linger about COVID-19 vaccines. If immunized individuals still have to wear masks, vaccine uptake will be further disincentivized.

For those vaccines that receive an EUA, the coming challenge will be to meet demand. The United States, Britain, France and Germany have already grabbed hundreds of millions of doses via advance purchase agreements, but COVAX - a global initiative coordinated by Gavi, the Coalition for Epidemic Preparedness Initiative (CEPI) and the World Health Organization, which now includes China as well as another 170 nations - aims to obtain two billion doses of vaccines by the end of 2021 for low-to-middle income countries.

A CEPI survey estimates that global manufacturing capacity could produce two to four billion doses next year. As different facilities ramp up production, supply-chain bottlenecks for glass vials, pressure on fill and finish facilities and shortages of materials like bioreactors or adjuvants are likely to arise.

The public may also be surprised to find that all vaccines are not equal. It may not realize that RNA and viral vector vaccines those most advanced in clinical development - are the least suitable for large-scale manufacture, storage and distribution and that they carry a greater technology risk. As no one has ever manufactured RNA vaccines with liposomal nanoparticles at this scale before - nor a viral vector vaccine - rollout might hit snags that tried and tested platforms (like inactivated or recombinant subunit vaccines) can avoid. The RNA vaccines also have onerous storage requirements (Pfizer/BioNtech's product must be kept at $-70{ }^{\circ} \mathrm{C}$, Moderna's at $-20{ }^{\circ} \mathrm{C}$ ). They are unlikely to be suitable for doctor's offices or pharmacies in developed nations, let alone in developing countries. Why not get that message out there now to offset disappointment later?

Perhaps the trickiest dilemma, however, will be prioritizing who receives the vaccine first. A recent US National Academy of Medicine report suggests that personal vulnerabilities (comorbidities and age) and social vulnerabilities (high-risk work settings, use of public transportation, and crowding or multiple generations at home) should determine allotment. Vaccine distribution would proceed in distinct phases: first, healthcare workers, care workers, first responders and those with pre-existing conditions; then essential workers, school teachers and people in prison (because of close quarters); and then everyone else. This is likely to be controversial. During the 2009 H1N1 influenza pandemic, for example, an association of bread makers argued that its workers were essential. We should expect wrangling about who qualifies to be in what phase.

The global distribution of coronavirus vaccines will be the defining public health challenge of 2021. There will be snafus. There will be delays. But whatever happens, an early and coordinated health communication campaign on various media is needed to drive uptake in vulnerable groups and engage diverse stakeholders in ethnic communities. Vaccines should be positioned alongside hand-washing, social distancing, masking and isolation as part of the pandemic solution, not a panacea to immediately end it. Governments need to start informing the public now by being transparent and open about where vaccines actually are - and effectively communicating their plans for distributing them.

Published online: 27 October 2020

https://doi.org/10.1038/s41587-020-0743-5 